\title{
Highly Soluble Tris-Hydroxypyridonate Gd(III) Complexes with Increased Hydration Number, Fast Water Exchange, Slow Electronic Relaxation, and High Relaxivity ${ }^{1}$
}

\author{
Eric J. Werner ${ }^{\dagger}$, Stefano Avedano ${ }^{\ddagger}$, Mauro Botta $^{\ddagger}$, Benjamin P. Hay $§$, Evan G. Moore ${ }^{\dagger}$, \\ Silvio Aime ${ }^{\|}$, and Kenneth N. Raymond ${ }^{\dagger}$ \\ tCollege of Chemistry, University of California, Berkeley, CA 94720-1460 \\ ‡Dipartimento di Scienze dell' Ambiente e della Vita, Università del Piemonte Orientale "A. \\ Avogadro", Alessandria, Italy \\ $\S$ Chemical Sciences Division, Pacific Northwest National Laboratory, Richland, WA 99352 \\ "Dipartimento di Chimica I.F.M., Università di Torino, Torino,Italy
}
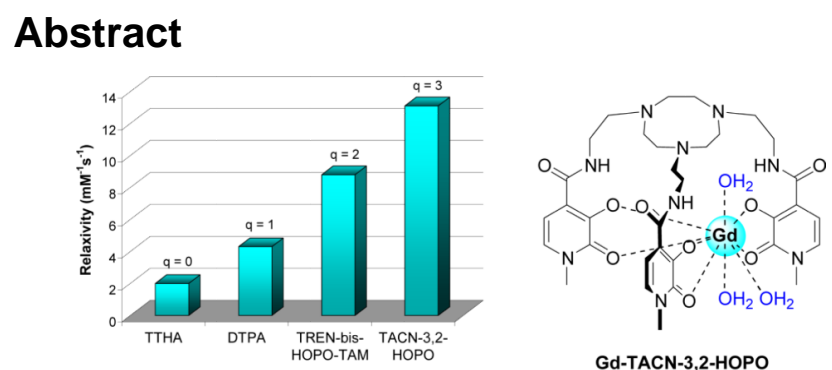

The design, synthesis, and relaxivity properties of highly soluble TACN-capped trishydroxypyridonate $\mathrm{Gd}(\mathrm{III})$ complexes are presented. Molecular mechanics modeling was used to help design a complex capable of possessing three water molecules in the inner metal coordination sphere, an attractive property for high-relaxivity MRI contrast agents. The measured relaxivities of 13.1 and $12.5 \mathrm{mM}^{-1} \mathrm{~s}^{-1}(20 \mathrm{MHz}, 298 \mathrm{~K})$ for two TACN-capped complexes are among the highest known relaxivities of low-molecular weight Gd complexes and are consistent with three coordinated waters, an extremely fast water exchange rate and long electronic relaxation time. Luminescence measurements to confirm the number of coordinated water molecules for the first time in the HOPO series are also discussed.

\begin{abstract}
Magnetic Resonance Imaging (MRI) is a powerful diagnostic technique in modern medicine, and the emergence of new instrumentation and applications invites the production of contrast agents with enhanced imaging capabilities. Contrast agents are evaluated on the basis of their proton relaxivities $\left(r_{1 \mathrm{p}}\right)$, and current values for commercial agents (based on poly-(aminocarboxylate) ligands) are small $\left(r_{1 \mathrm{p}}=4-5 \mathrm{mM}^{-1} \mathrm{~s}^{-1} ; 20 \mathrm{MHz}\right.$ and $\left.298 \mathrm{~K}\right)$ compared to what is theoretically possible. ${ }^{2,3}$ To obtain the high relaxivities predicted by theory, there are several parameters that must be optimized, including: $q$, the number of bound waters, $\tau_{\mathrm{m}}$, the mean water residence time, $\tau_{\mathrm{s}}$, the metal electronic relaxation time,
\end{abstract}

Correspondence to: Kenneth N. Raymond.

raymond@socrates.berkeley.edu .

Supporting Information Available: Detailed synthesis and characterization of $\mathbf{3}$ and of TACN-1,2-HOPO, temperature dependence of the paramagnetic contribution to the water ${ }^{17} \mathrm{O}$ NMR transverse relaxation rate $\left(R_{2} \mathrm{p}\right)$ for $\mathbf{3}$, and details of the luminescence and stability measurements. This information is available free of charge via the internet at http://pubs.acs.org. 
and $\tau_{\mathrm{R}}$, the rotational correlation time. ${ }^{2,4}$ While improvement of any one of these key parameters can yield optimized relaxivities in the 20-40 MHz frequency range for lowmolecular weight complexes, ${ }^{5}$ the goal remains to optimize all parameters to attain high relaxivity at the higher magnetic field strengths of current and future clinical MRI scanners. Here we report stable, soluble complexes with optimal hydration, water exchange, and electronic relaxation properties, representing major advances toward that goal.

In 1995, a Gd complex was described that showed promise as a contrast agent, Gd(TREN-1Me-3,2-HOPO $\left(\mathrm{H}_{2} \mathrm{O}\right)_{2}\left(\mathbf{1}\right.$, Figure 1). ${ }^{6}$ The $r_{1 \mathrm{p}}$ value of this hexadentate hydroxypyridinone(HOPO) based complex was measured as twice that of commercial agents due largely to the complex's increased number of bound water molecules ( $q$ of 2 as compared to 1 for commercial agents). Importantly, the stability of this complex (a critical factor for in vivo use $^{7}$ ) is higher than that of clinical agents despite the lower ligand denticity and increase in $q$. Since this initial report, a series of complexes, all based on the tripodal TREN (tris-(2aminoethyl)-amine) scaffold, has been prepared in an attempt to optimize the proton relaxation parameters and increase aqueous solubility. ${ }^{8}$ Those complexes that show the most promising relaxometric and solubility properties rely on the TREN-capped heteropodal bisHOPO-TAM design (2, Figure 1). For example, stabilization of the nine-coordinate, $q=3$ ground state was recently reported in the TREN-bis-HOPO-TAM system by incorporating substituents on the terephthalamide (TAM) chelator capable of hydrogen bonding interactions with a third inner-sphere water as suggested by relaxivity and NMRD refinements. ${ }^{9}$ While such an approach shows the potential for $q=3$ complexes of higher relaxivity, the synthesis of the asymmetric bis-HOPOTAM compounds is arduous, requiring high-dilution, slow-addition reaction conditions for practical yields. It would be advantageous to generate soluble $q=3$ complexes without the need for such a design to produce high-relaxivity agents which optimize not only $q$, but the other relevant parameters such as water exchange and electronic relaxation in a more straightforward manner.

The work reported herein describes a new approach toward stable, high-relaxivity trisHOPO complexes by replacing TREN with a triazacyclononane (TACN) derivative as the ligand cap. The compounds of interest in this study, Gd-TACN-3,2-HOPO and GdTACN-1,2-HOPO ( $\mathbf{3}$ and $\mathbf{4}$, Figure 1) were proposed and complex $\mathbf{3}$ was modeled using previously developed molecular mechanics techniques ${ }^{10}$ to predict the effect of the larger TACN capping scaffold on the number of inner-sphere waters molecules. Two methods were used to predict $q$, and both the exposed metal surface area $\left(8.2 \AA^{2}\right.$, threshold $\left.=6.0 \AA^{2}\right)$ and strain energy of adding inner-sphere waters $(2.7 \mathrm{kcal} / \mathrm{mol}$, threshold $=9.5 \mathrm{kcal} / \mathrm{mol})$ strongly support a $q=3 \mathrm{Gd}$ complex. In addition to the potential effect on $q$, utilization of a TACN capping structure should afford Gd complexes of high aqueous solubility, a key parameter which has limited application of tris-HOPO complexes. Employing TACN as the ligand cap should thus enable more straightforward procedures to generate tris-HOPO ligands without requiring a TAM podand further derivatized with solubilizing substituents.

Synthesis of Gd-TACN-3,2-HOPO (3) is shown in Scheme S1 (see Supporting Information) and entails the amide coupling of thiazolide-activated 3,2-HOPO precursor (5) with the primary amines of derivatized TACN $(\mathbf{6})$ synthesized from a published procedure. ${ }^{11}$ Deprotection under acidic conditions yields the ligand which is next complexed with gadolinium to form 3 . TACN-1,2-HOPO is synthesized in a similar manner, replacing 5 with thiazolide-activated 1,2-HOPO (Scheme S2). The effect of the TACN cap on aqueous solubility is dramatic, as more than a 1000-fold increase in solubility over that observed for the parent Gd-TREN-tris-3,2-HOPO complex (1) was determined for $3(\sim 100 \mathrm{mM})$.

To directly assess the influence of the TACN scaffold on the number of bound water molecules, the $\mathrm{Eu}(\mathrm{III})$ complex of TACN-1,2-HOPO was prepared for luminescence 
lifetime measurements as it was recently determined that 1,2-HOPO efficiently sensitizes Eu luminescence. ${ }^{12}$ This measurement also represents the first direct measurement of the solution state $q$ value in the HOPO family of MRI agents, which until now had been assumed from relaxivity values and NMRD profile fittings. Measurement of the lifetimes in $\mathrm{H}_{2} \mathrm{O}$ and $\mathrm{D}_{2} \mathrm{O}$ were performed, and application of the modified Horrocks equation ${ }^{13}$ yielded a $q$ value of $2.9 \pm 0.5$ at $\mathrm{pH} 7.0$, in excellent agreement with the molecular modeling results and verifying a 9-coordinate metal geometry for TACN-capped hexadentate HOPO ligands.

Relaxivity measurements were obtained for $\mathbf{3}$ and $\mathbf{4}$ to determine if the complexes deliver the predicted relaxation enhancements. Relaxivities of 13.1 and $12.5 \mathrm{mM}^{-1} \mathrm{~s}^{-1}(20 \mathrm{MHz}, 298$ $\mathrm{K}, \mathrm{pH} 7$ ) were obtained for $\mathbf{3}$ and $\mathbf{4}$, respectively, and are among the highest known values of low-molecular weight mononuclear Gd complexes. The NMRD (Nuclear Magnetic Relaxation Dispersion) profiles of each complex were obtained and analyzed, fixing $q$ to a value of 3 (Figure 2). Noteworthy is the consistent high relaxivity with increasing field strength, a feature not frequently observed for aminocarboxylate-based compounds. The high solubility of $\mathbf{3}$ near neutral $\mathrm{pH}$ also allowed for the direct assessment of the water exchange rate by variable temperature ${ }^{17} \mathrm{O} N \mathrm{NMR}$, giving a $\tau_{\mathrm{M}}$ value of ca. $2.0 \mathrm{~ns}$ which represents one of the fastest water exchange rates yet measured for a HOPO-based Gd complex. The optimal value of this parameter decreases with increasing field strength, passing from ca. $30 \mathrm{~ns}$ at $20-40 \mathrm{MHz}$ to ca. $2-4 \mathrm{~ns}$ at $80-100 \mathrm{MHz}$. Thus, the rate of water exchange of $\mathbf{3}$ is ideal for the preparation of macromolecular derivatives of enhanced relaxivity at relevant field strengths. In addition to an increase in hydration number and water exchange, the $\Delta^{2}$ values obtained from the NMRD fits (Table 1) are the lowest yet determined for any HOPO-based Gd complex and suggest a longer electronic relaxation time, another desirable feature for high-relaxivity agents. The increase in this parameter may be due to the rigidity and symmetry imparted to the metal center by the TACN cap, as was seen in other Gd complexes employing the TACN structure. ${ }^{14-16}$ Analysis of the emission spectrum of Eu-TACN-1,2-HOPO (see Supporting Information) indicates that the complex possesses the $\mathrm{D}_{3 \mathrm{~h}}$ geometry at the metal center which is of higher symmetry than the $\mathrm{C}_{2 \mathrm{v}}$ geometry of the parent di-aquo TREN-capped complex. It is known that higher symmetry increases $\tau_{\mathrm{s}}$ which would further augment relaxivity. ${ }^{2}$

In summary, this work presents the design, synthesis, and characterization of soluble TACNcapped tris-HOPO Gd(III) complexes with optimal properties for high relaxivity. Molecular modeling studies indicated that the TACN cap facilitates a nine-coordinate $q=3$ ground state, and relaxivity studies combined with, for the first time, luminescent determination of $q$ revealed a successful design strategy. To further probe the viability of these compounds as imaging agents, stability and relaxivity studies of these compounds under more physiologically relevant conditions have commenced. A preliminary pGd of 18.7 for TACN-3,2-HOPO has been determined (see Supporting Information), and the relaxivity in serum was measured as $17 \mathrm{mM}^{-1} \mathrm{~s}^{-1}$. This pGd value compares well with those of commercial agents, and the persistent high relaxivity of $\mathbf{3}$ in serum indicates that anion/ protein binding, which could potentially replace bound waters and decrease relaxivity, does not occur. The high relaxivity of TACN-capped complexes such as $\mathbf{3}$, combined with its high stability, solubility, fast water exchange, and slow electronic relaxation, presents exciting implications for usage with high-field MRI scanners and imaging applications such as site-specific targeted agents. Future work includes the attachment to larger structures, such as dendrimers or proteins, to slow molecular tumbling and achieve even higher relaxivity.

\section{Supplementary Material}

Refer to Web version on PubMed Central for supplementary material. 


\section{Acknowledgments}

E.J.W and K.N.R acknowledge support from the NIH (grant HL69832) and NATO (PST.CLG.980380). B.P.H. acknowledges support from the Environmental Management Science Program, Office of Science, U.S. Department of Energy (grant 73759).

\section{References}

1. Paper \#20 in the series High Relaxivity Gadolinium MRI Agents. For the previous paper see: Pierre VC, Botta M, Aime S, Raymond KN. Inorg. Chem. 2006; 45:8355-8364. [PubMed: 16999435]

2. Caravan P, Ellison JJ, McMurry TJ, Lauffer RB. Chem. Rev. 1999; 99:2293-2352. [PubMed: 11749483]

3. Aime S, Botta M, Fasano M, Geninatti Crich S, Terreno E. Coord. Chem. Rev. 1999; 185-6:321333.

4. Tóth, E.; Helm, L.; Merbach, AE. The Chemistry of Contrast Agents in Medical Magnetic Resonance Imaging. Wiley; Chichester: 2001. p. 45-119.

5. Messeri D, Lowe MP, Parker D, Botta M. Chem. Commun. 2001:2742-2743.

6. Xu J, Franklin SJ, Whisenhunt DW, Raymond KN. J. Am. Chem. Soc. 1995; 117:7245-7246.

7. Ranganathan RS, Raju N, Fan H, Zhang X, Tweedle MF, Desreux JF, Jacques V. Inorg. Chem. 2002; 41:6856-6866. [PubMed: 12470084]

8. Raymond KN, Pierre VC. Bioconjugate Chem. 2005; 16:3-8.

9. Pierre VP, Botta M, Aime S, Raymond KN. J. Am. Chem. Soc. 2006; 128:5344-5345. [PubMed: 16620097]

10. Hay BP, Werner EJ, Raymond KN. Bioconjugate Chem. 2004; 15:1496-1502.

11. Tei L, Baum G, Blake AJ, Fenske D, Schröder M. J. Chem. Soc., Dalton Trans. 2000:2793-2799.

12. Moore EG, Xu J, Jocher CJ, Werner EJ, Raymond KN. J. Am. Chem. Soc. 2006; 128:1064810649. [PubMed: 16910637]

13. Beeby A, Clarkson IM, Dickins RS, Faulkner S, Parker D, Royle L, de Sousa AS, Williams JAG, Woods M. J. Chem. Soc., Perkin Trans. 2. 1999:493-503.

14. Chatterton N, Gateau C, Mazzanti M, Pécaut J, Borel A, Helm L, Merbach A. Dalton Trans. 2005:1129-1135. [PubMed: 15739017]

15. Fries PH, Gateau C, Mazzanti M. J. Am. Chem. Soc. 2005; 127:15801-15814. [PubMed: 16277523]

16. Nonat A, Gateau C, Fries PH, Mazzanti M. Chem. Eur. J. 2006; 12:7133-7150. 


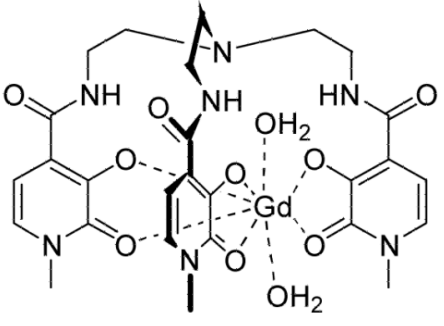

1

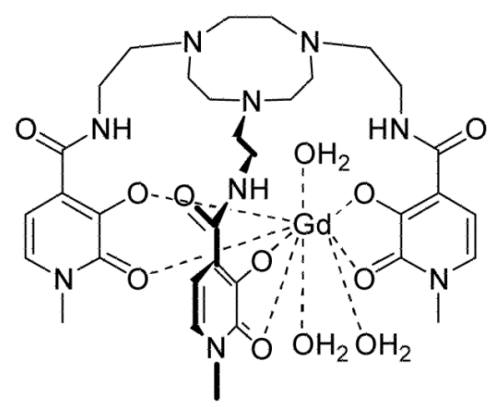

3
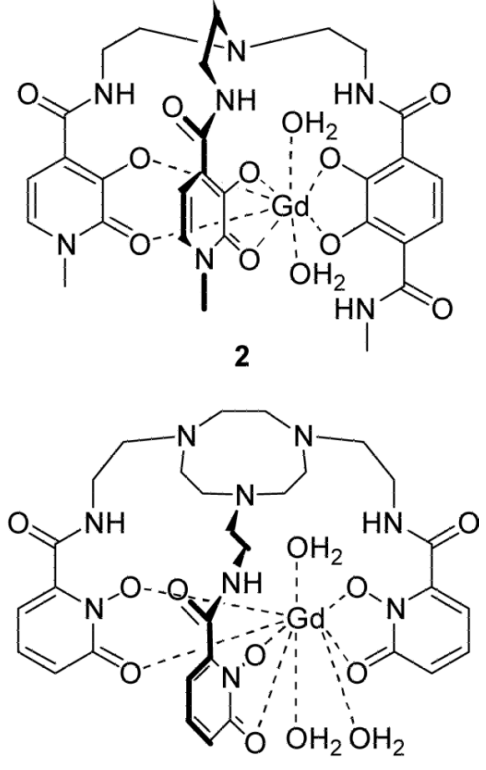

4

Figure 1.

Gd-TREN-1-Me-3,2-HOPO (1); Gd-TREN-bis-(1-Me-3,2-HOPO)-TAM (2); GdTACN-3,2-HOPO (3); Gd-TACN-1,2-HOPO (4). 


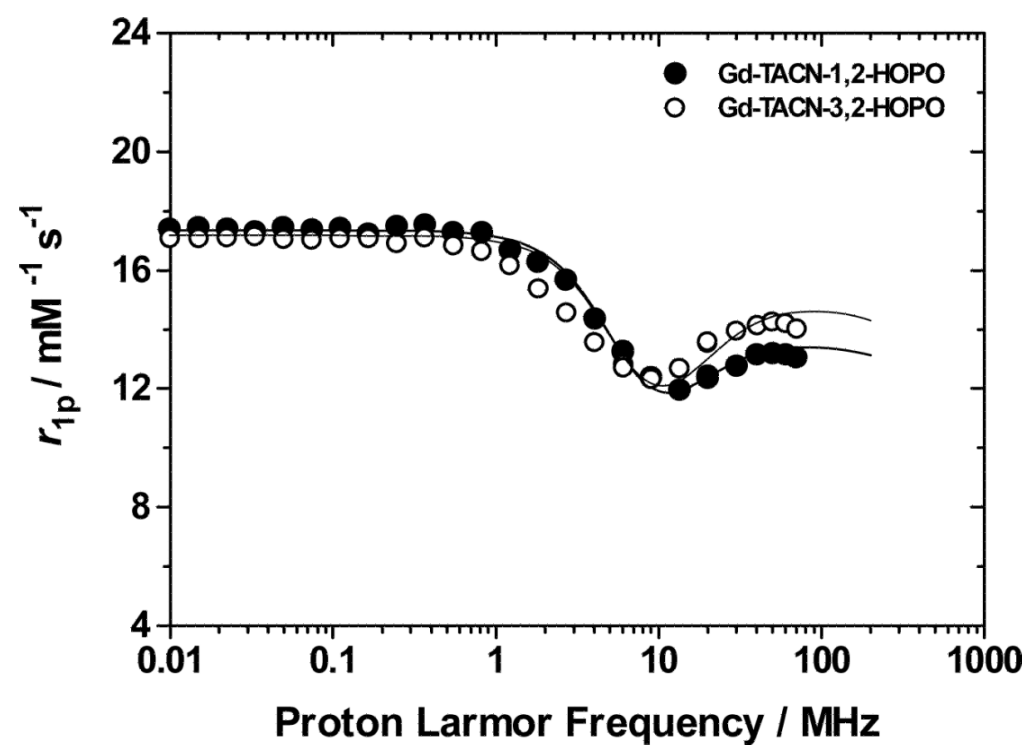

Figure 2.

1/T 1 NMRD profiles of $3(\mathrm{pH} \mathrm{6.1)}$ and $4(\mathrm{pH} \mathrm{7.1)}$ at $298 \mathrm{~K}$. 
Table 1

Relevant parameters obtained from the fitting of ${ }^{1} \mathrm{H}$ NMRD profiles ${ }^{a}$ and ${ }^{17} \mathrm{O}$ data.

\begin{tabular}{|c|c|c|}
\hline & $\mathbf{3}^{\boldsymbol{b}}$ & $\mathbf{4}^{\boldsymbol{c}}$ \\
\hline$\Delta^{2}\left(\mathrm{~s}^{-2} ; \times 10^{19}\right)$ & $6.3 \pm 0.4$ & $5.8 \pm 0.3$ \\
$E_{\mathrm{V}}\left(\mathrm{kJ} \mathrm{mol}^{-1}\right)$ & $1.0^{d}$ & $/$ \\
$\tau_{\mathrm{R}}(\mathrm{ps})$ & $150 \pm 3$ & $130 \pm 5$ \\
$E_{\mathrm{R}}\left(\mathrm{kJ} \mathrm{mol}^{-1}\right)$ & $18^{d}$ & $/$ \\
$\tau_{\mathrm{M}}^{298}(\mathrm{~ns})$ & $2.0 \pm 0.2^{d}$ & $2^{e}$ \\
$\Delta H_{\mathrm{M}}\left(\mathrm{kJ} \mathrm{mol}^{-1}\right)$ & $6.7 \pm 0.9^{d}$ & $/$ \\
$\Delta S_{\mathrm{M}}\left(\mathrm{J} \mathrm{mol}^{-1} \mathrm{~K}^{-1}\right)$ & $-55.6 \pm 3.1^{d}$ & $/$ \\
\hline
\end{tabular}

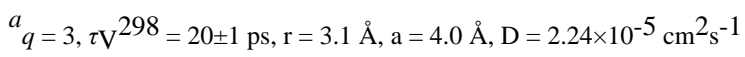

$b_{\mathrm{pH}} 6.1$

${ }_{\mathrm{pH}} 7.1$

$d_{\text {obtained from the fitting of VT }}{ }^{17} \mathrm{O}$ NMR data

$e_{\text {fixed. }}$ 